\title{
Maintenance therapy with oral etoposide following first-line docetaxel-cisplatin chemotherapy in metastatic non-small cell lung cancer patients
}

\author{
Meiyu Fang, Shengye Wang, Yabing Zheng, Xiangmin Kong, Liyan Gong, Youhao Qiu, \\ Yazhen Zhao and Weimin Mao
}

Zhejiang Thoracic Oncology Institute, Zhejiang Province Cancer Hospital, Zhejiang Cancer Center, No. 38 Guangji Road, Banshanqiao District, Hangzhou 310022, People's Republic of China.

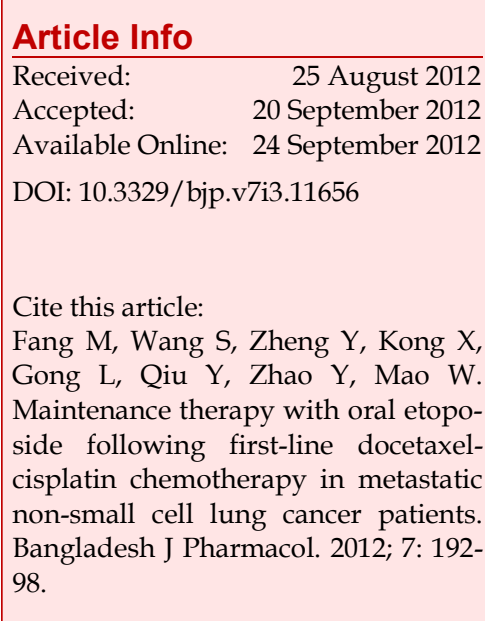

\section{Abstract}

The present study evaluated the efficacy and safety of maintenance therapy with oral etoposide following first-line docetaxel-cisplatin chemotherapy in patients with metastatic non-small cell lung cancer. The overall response rate in the docetaxel-cisplatin phase was $30.4 \%$, with complete response of 0 , partial response of 28, stable disease of 39. Maintenance chemotherapy with oral etoposide was given in 67 patients. No complete response was observed, but 10 patients presented with partial response and 45 with stable disease . The median time to progression was 5.0 months; median overall survival time was 10 months and the 1 -year survival rate was $41 \%$ during the maintenance therapy. Therapy with oral etoposide was well tolerated with the hematologic toxicities, fatigue and oral mucositis being the most frequent side effects. Docetaxel-cisplatin chemotherapy followed by maintenance therapy with oral etoposide was well tolerated and moderately active against metastatic metastatic non-small cell lung cancer.
\end{abstract}

\section{Introduction}

Lung cancer has been the most common cancer in terms of both incidence and mortality worldwide (Jemal et al., 2009. 70-75\% of all lung cancers are non small-cell lung cancer (NSCLC) with two-thirds presenting with locally advanced or metastatic disease at diagnosis.

Treatment for these patients includes chemotherapy, radiotherapy, molecular targeted therapy, and best supportive care. Etoposide, a derivative of podophyllotoxin, is an important chemotherapeutic agent that is a phase-specific, schedule-dependent semi-synthetic epipodophyllotoxin with modest activity against metastatic NSCLC when used alone (Pedersen and Hansen, 1983). Etoposide has well-known effectiveness in the NSCLC. It is possible that to exploit the 'continuing effect' of prolonged low dose oral etoposide could produce efficient anti-tumor effect. Oral agents with few side effects are appealing in the 2nd or 3rd line settings where patients are usually in a less favorable condition. Given the favorable efficacy of etoposidecisplatin combination in concurrent chemotherapy/ radiotherapy (Albain et al., 2002), maintenance therapy with oral etoposide prior to progression may improve therapeutic efficacy in patients with metastatic NSCLC in the absence of prior exposure to etoposide.

We hypothesized that full dose platinum doublet administered at the beginning of the cycle could induce the tumor debulking, and subsequently a prolonged administration of low dose oral etoposide could produce a more efficient antitumor effect. In the present study, we investigated if oral etoposide for maintenance chemo- 


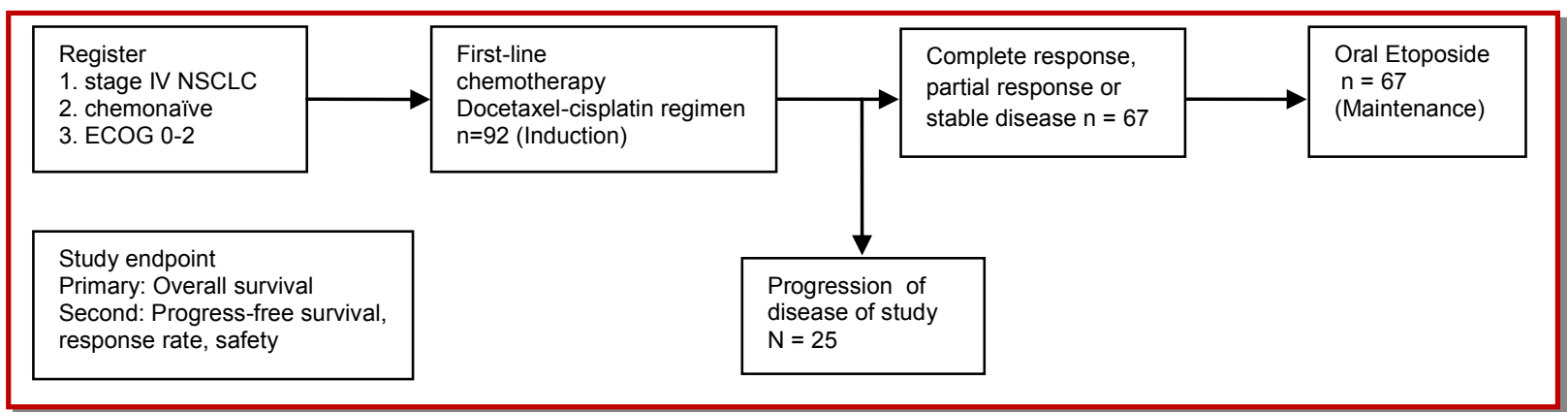

Figure 1: Diagram of study. Docetaxel/cisplatin. docetaxel $\left(75 \mathrm{mg} / \mathrm{m}^{2}\right.$, day 1) plus cisplatin $\left(75 \mathrm{mg} / \mathrm{m}^{2}\right.$, day 1$)$. After 4 cycles of induction treatment, patients without progression of disease received oral etoposide for maintenance therapy

therapy could improve the therapeutic efficacy following the standard induction therapy with platinumbased doublets (docetaxel-cisplatin) in patients with advanced NSCLC.

\section{Materials and Methods}

\section{Subjects}

The present study was to investigate the PFS of patients with metasatistic NSCLC who received oral etoposide for maintenance chemotherapy plus BSC. These patients had no progression of disease during the firstline 4 cycles of chemotherapy with docetaxel-cisplatin, and to determine the overall survival, objective tumor response rate and toxicity of this regimen.

\section{Patient selection}

Patients with histologically confirmed stage IV NSCLC were eligible for this study. Patients were required to have no prior chemotherapy or thoracic radiotherapy, but those experiencing post-operative recurrence were eligible. Additional inclusion criteria included a performance status of 2 or less according to World Health Organization criteria, 18-75 years or less, no brain metastasis, no other cancers, no interstitial pneumonitis, no severe cardiac diseases, no cirrhosis, adequate bone marrow reserve (white blood count $\geq 3 \times 10^{9} / \mathrm{L}$, absolute neutrophil count $\geq 1.5 \times 10^{9} / \mathrm{L}$, platelet count $\geq 100 \times$ $10^{9} / \mathrm{L}$ ) and a creatinin clearance rate of $>50 \mathrm{~mL} / \mathrm{min}$.

All eligible patients were evaluated before registration by physical examination, chest $\mathrm{X}$-ray; computer tomography of the chest, upper abdomen and brain, abdominal ultrasonography, fiberoptic bronchoscopy followed by biopsies and detection of complete blood cell count and creatinine. Written informed consent was obtained from each patient before study.

\section{Treatment}

The eligible patients received two courses of chemotherapy: Induction phase and maintenance phase. In the induction phase, patients received 4 cycles of induction chemotherapy with docetaxel and cisplatin at a 21-day cycle. The regimen consisted of docetaxel (Taxoteres, Aventis Pharma) at $75 \mathrm{mg} / \mathrm{m}^{2}$ in $250 \mathrm{~mL}$ of $5 \%$ dextrose administered intravenously over 1 hours. This was followed by intravenous cisplatin at $75 \mathrm{mg} / \mathrm{m}^{2}$ in 250 $\mathrm{mL}$ of normal saline over $30-60 \mathrm{~min}$ on day 1 . The hydration schedule included 1-1 normal saline with 20 mmol potassium chloride and $16 \mathrm{mmol}$ magnesium sulfate given over 2 hours before docetaxel infusion and $1.510 .45 \%$ saline $/ 2.5 \%$ dextrose over 12 hours after cisplatin infusion on day 1 . In addition, $180 \mathrm{~mL}$ of $20 \%$ mannitol was given over 2.5 hours immediately before and after cisplatin infusion on day 1. Chemotherapy was repeated every 3 weeks, unless otherwise contraindicated, for a total of 4 cycles.

Following induction treatment, patients who achieved disease controlled (complete response + partial response + stable disease) were enrolled for the maintenance chemotherapy: Oral etoposide (Bristol-Myers Oncology) administered at $35 \mathrm{mg} / \mathrm{m}^{2} /$ day for 21 consecutive days every 28 days plus BSC. The dose of etoposide was reduced by $25-50 \%$ for Grade 4 toxicity. Treatment was delayed for 1 week if neutrophils were less than $1,500 / \mu \mathrm{L}$ or platelets less than $50,000 / \mu \mathrm{L}$. Maintenance chemotherapy began at the time of day 2128 from day 1 of the 4 th cycle of induction therapy. Maintenance therapy ended when the patients developed one of the pre-specified manifestations for discontinuation, including progression of disease. Patients who discontinued maintenance treatment in the absence of progression of disease continued to receive periodic tumor response evaluation. Once the patient presented with progression of disease, followup was performed every 90 days until death or the end of study. Information regarding anticancer systemic therapy, radiotherapy, and surgical intervention was also collected after treatment discontinuation. During the study, all patients received standard prophylactic dexamethasone, as specified in the docetaxel label. The whole protocol was approved by the ethics committee of Zhejiang Cancer Hospital, and the study was conducted in accordance with the ethical principles of 
the Declaration of Helsinki and good clinical practice.

\section{Evaluation of response and statistics}

The survival was assessed using the Kaplan-Meier method. RECIST method was used to evaluate the response rate, and toxicity to chemotherapy was graded according to NCI-CTC version 3. The Kaplan-Meier method was used for the analyses of overall survival and PFS time-to-event. The Cox proportional hazards model was used to estimate hazard rates with assigned treatment as the only covariate. Tumor response rate (partial response + complete response) and disease control rate (complete response + partial response + stable disease) were compared between arms using the Fisher's exact test. The tumor response to maintenance therapy was based on the last radiologic assessment before randomization as baseline. The tumor response to whole treatment (induction and maintenance) was based on the radiologic assessment before induction therapy as baseline. Statistical analyses were conducted using the SPSS version 13.0 for windows. A value of $p$ less than 0.05 was considered statistically significant.

\section{Results}

From October 2007 through March 2010, a total 92 patients were treated with docetaxel-cisplatin (Table I). A total of 348 cycles of chemotherapy with docetaxelcisplatin were administered to these patients (median: 4 cycles; range, $1-4$ cycles); 77 (83.7\%) patients received 4 cycles. The treatment in approximately $90 \%$ of cycles was performed at the initial dose of $75 \mathrm{mg} / \mathrm{m}^{2}$. Dose modification, mostly due to toxicity, was found in only $5 \%$ of cycles. Delayed treatment was noted in $9 \%$ of cycles. The treatment was frequently delayed between days 3 and 7. No patient experienced complete response, 28 patients $(30.4 \%)$ had partial response, and 39 patients $(42.4 \%)$ had stable disease. The overall response rate in the induction phase was 30.4\%. In addition, 25 patients discontinued the 1st line chemotherapy due to progression of disease (27.2\%) (Table II).

A total 67 patients experiencing disease control rate $(72.8 \%)$ were enrolled for the maintenance chemotherapy with oral etoposide (Table I). All received evaluation of tumor response and PFS (Table II). A total of 295 chemotherapy cycles delivered, range from 1 to 12 . Twenty one patients (31.3\%) were older than 70 years and $5(7.2 \%)$ younger with a poor performance status. The mean number of cycles in the etoposide arm was 4.4 cycles. None of the 67 patients $(0 \%)$ achieved complete response. Partial response was seen in 10 of 67 patients $(14.9 \%)$, and $45(67.2 \%)$ patients achieved stable disease. The disease control rate was $82.1 \%$ (55/67). Figure 1 shows the PFS among these
Table I

Baseline characteristics of 92 patients with meta-

static NSCLC in the trial with oral etoposide

Characteristics

$\mathrm{n}$

$\begin{array}{ccc} & \text { Baseline } & \text { Etoposid } \\ \text { Total } & 92 & 58\end{array}$

Median age (Years)

$<70$

63

46

$\geq 70$

29

21

Gender

Male

Female

Smoking status

Current/former

$71 \quad 48$

Never

21

ECOG performance status

0-1

2

Histology

Squamous cell cancer

39

Adenocarcinoma

Large cell cancer

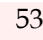

36

3

25

patients. The median PFS was 5 months.

The most common grade 3 and 4 hematologic toxicities in the first line chemotherapy were neutropenia (25/92, $27.2 \%)$, thrombocytopenia (9/92, 9.8\%), and anemia (5/92, 5.4\%; Table III)). The most common grade 3 and 4 nonhematologic toxicities in the docetaxel-cisplatin treatment were nausea, vomiting, and alopecia. The most grade 1 and 2 toxicities were neuropathy and diarrhea.

Toxicities in the maintenance therapy with oral etoposide in the 67 patients are presented in Table III. The main toxicity was hematologic. Grade I-II hematological toxicity was the most common, and observed in 28 patients $(41.8 \%)$. Grade I-II asthenia, nausea, vomiting and alopecia were also noted. Grade 3 and 4 leukopenia and thrombocytopenia were observed in 9 patients $(13.4 \%)$ and 1 patient $(1.5 \%)$, respectively. No patients experienced grade 3 febrile neutropenia. One case of non-neutropenic fever and three cases of pneumonia were found. These patients recovered upon antibiotic and anti-mycotic treatment. The most common grade 3 and 4 non-hematologic toxicities in the mainteinance treatment were fatigue $(6.2 \%)$ and oral mucositis. There was no observed grade 3 or 4 neuropathy in the maintenance treatment. Toxicity related death was not found. 
Table II

Tumor response after induction chemotherapy (docetaxel and cisplatin) and 2 months of treatment with oral etoposide

\begin{tabular}{|lcc|}
\hline Response & $\mathrm{n}$ & $\mathrm{n}$ \\
\cline { 2 - 3 } & $\begin{array}{c}\text { Docetaxel and cis- } \\
\text { platin }\end{array}$ & Etoposide \\
Complete response & 0 & 0 \\
Partial response & 28 & 10 \\
Stable disease & 39 & 45 \\
Progression of disease & 25 & 12 \\
Overall response & 28 & 10 \\
Disease control rate & 67 & 55 \\
\hline
\end{tabular}

\section{Discussion}

Palliative chemotherapy is still the standard first-line treatment for stage IV NSCLC patients with good performance status. This generally comprises one of several equally effective cytotoxic doublets, including a platinum analogue combined with either vinorelbine, gemcitabine, taxane, docetaxel or pemetrexed for patients with non-squamous cell cancer (Schiller et al., 2002; Scagliotti et al., 2008). Numerous efforts have been made to improve the efficacy of first-line chemotherapy for the advanced NSCLC. The standard platinum-based doublets have comparable efficacy, but slightly different toxicity profiles (Schiller et al., 2002). Some superior treatment outcomes were associated with specific patients or disease characteristics. In a phase III study in which patients with advanced NSCLC were studied, a preplanned subgroup analysis showed a difference in histological response (Scagliotti et al., 2008). Patients with non-squamous histology had statistically superior overall survival with cisplatin/ pemetrexed than with cisplatin/gemcitabine, which was also supported by additional retrospective analyses in phase II studies (Ohe et al., 2008). The difference in response rate may be related to the level of thymidylate synthase, which is the primary target of the pemetrexed. In different cancers, preclinical data support the hypothesis that over-expression of thymidylate synthase correlates with reduced sensitivity to pemetrexed (Giovannetti et al., 2005), and high thymidylate synthase expression level was associated with poor outcome of patients treated with antifolates.

Molecular targeted therapy has recently been examined as a means to improve the efficacy of first-line chemotherapy in advanced NSCLC. The epidermal growth factor receptor tyrosine kinase inhibitors erlotinib and gefitinib were not shown to improve the efficacy when added to the platinum doublet, regardless of the epidermal growth factor receptor status (Giaccone, 2005; Hirsch et al., 2011). However, they are effective, as single agents, for patients with EGFR mutations (Fukuoka and Wu, 2011). Cetuximab and bevacizumab, monoclonal antibodies against epidermal growth factor receptor and vascular endothelial growth factor, respectively, have shown to improve the survival when added to standard first-line chemotherapy (Pirker et al., 2006; Sandler et al., 2006) . However, cetuximab is not currently approved for NSCLC treatment, and the toxicity limits the use of bevacizumab in the treatment of non-squamous cancer. Therefore, the platinum-based chemotherapy remains the main treatment of choice for many patients with NSCLC. In addition to the development of novel agents and combinations for the first-line treatment of advanced NSCLC, efforts have focused on the improvement of efficacy and tolerability via altering the treatment schedule. One strategy to improve the outcome is to utilize the maintenance therapy. Maintenance therapy in NSCLC is defined as the continuation of an active treatment until progression of disease in patients who have demonstrated at least a non-progressing status following the first-line

\section{Table III}

Toxicity to induction chemotherapy and maintenance therapy with oral etoposide according to NCI-CTC

\begin{tabular}{|lcccc|}
\hline & \multicolumn{4}{c|}{ Number of patients } \\
\cline { 2 - 5 } & \multicolumn{1}{c|}{ Induction chemotherapy } & \multicolumn{2}{c|}{ Maintenance therapy } \\
& $\begin{array}{c}\text { Toxicity grade } \\
\text { G1 + }\end{array}$ & Toxicity grade & Toxicity grade & $\begin{array}{c}\text { Toxicity grade } \\
\text { G3 }+4\end{array}$ \\
\hline Neutropenia & 49 & 25 & 20 & 9 \\
Anemia & 13 & 5 & 7 & 1 \\
Thrombocytopenia & 11 & 9 & 6 & 0 \\
Nausea and vomiting & 31 & 10 & 5 & 0 \\
Diarrhea & 20 & 6 & 2 & 0 \\
Alopecia & 43 & 47 & 7 & 0 \\
Stomatitis & 26 & 8 & 14 & 12 \\
Fatigue & 33 & 5 & 13 & 9 \\
\hline
\end{tabular}


chemotherapy. This concept originates from haematooncology and was used in the treatment of NSCLC referring to the immediate therapy after 4-6 cycles of standard first-line (or "induction") chemotherapy.

"Continued maintenance therapy" is when one or more of the agents from the first line setting is continued after a fixed number of cycles of first-line therapy, and "switch maintenance therapy" refers to the use of a different drug. It is administered for either a defined number of cycles or until progression of disease. The goals of maintenance therapy are to improve the overall survival, delay the tumor progression, maintain or improve the quality of life and minimize the side effects, especially cumulative toxicities. A reduction in cancer-related symptoms such as anorexia or fatigue should be balanced against the toxicities of maintenance chemotherapy. The cost-benefit ratio is also important. Theoretically, the maintenance therapy has the advantage of allowing patients to continue chemotherapy while the cancer burden is low (compared to treatment after progression of disease). One of the reasons in favor of maintenance therapy is that patients are generally in a better overall condition if therapy is initiated as early as possible. Evidence suggests that, following a platinum-containing induction, maintenance therapy using the non-platinum agent in the induction regimen enables the identification of patients most likely to benefit from the maintenance treatment. One of the phase III studies on the maintenance therapy examined the efficacy of maintenance therapy with gemcitabine following 4 cycles of induction therapy with cisplatin/gemcitabine (Brodo-wicz et al., 2006). Patients without progression of disease following induction therapy randomly received either gemcitabine or BSC. The median time to progression of disease was significantly longer in patients receiving gemcitabine treatment. Additionally, a significantly longer overall survival time was found in patients with a high baseline Karnofsky performance status $(>80)$ who were treated with gemcitabine when compared with those receiving BSC, whereas the difference in survival was not statistically significant between patients with a low baseline performance status.

According to the Goldie and Coldman hypothesis, the proportion of chemotherapy-resistant cells in a cancer increases over time. Thus, administration of new drugs as early as possible after first-line therapy has a potential advantag (Goldie and Coldman, 2008). By using a drug different from those used in the induction regimen for maintenance therapy, patients are exposed to a chemotherapeutic agent with a different pharmacological action. A phase III study compared docetaxel given immediately after 4 cycles of carboplatin/gemcitabine (as a maintenance therapy) with docetaxel administered after progression of disease (as a second-line therapy) (Fidias et al., 2008). Statistically significant improvements in progression-free survival (PFS) and overall survival were observed in patients who received docetaxel as a maintenance therapy.

Treatment with docetaxel has been found to improve the long term survival of metastatic NSCLC patients; but the median survival is only about 4-5 months. The therapeutic efficacy of this strategy can be improved by additional maintenance therapy, which has recently been investigated in a series of studies. Maintenance chemotherapy of NSCLC can be defined as continuation of an active treatment until progression of disease in patients who have demonstrated in at least a nonprogressing status following the first-line chemotherapy, and this therapy continues typically until the occurrence of unacceptable toxicity or progression of disease. First-line chemotherapy is usually limited to 4 cycles. Chemoresistance is common in NSCLC. All NSCLC patients will eventually develop resistance to the chemotherapeutic agents to which they are exposed, even in those with a good initial response, and most patients have to receive two or three line therapy.

In an effort to improve the effectiveness of first-line cisplatin/docetaxel therapy for metastatic NSCLC and to avoid the development of chemoresistance, oral etoposide was used for maintenance chemotherapy immediately following 4 cycles of induction therapy, and whether oral etoposide for maintenance therapy following 4 cycles of cisplatin/docetaxel induction therapy provides an additional benefit on the efficacy without substantially affecting the safety was investigated in the present study.

Our results show that first-line docetaxel-cisplatin therapy was well tolerated, with myelosuppression being the major cause of toxicities and no patients in the docetaxel-cisplatin phase discontinued the treatment due to the toxicities. The overall response in this cohort was $(30.4 \%)$, with SD in $42.4 \%$ of patients. The response and toxicities were consistent with those in previous reports on docetaxel-cisplatin as first line chemotherapy for metastatic NSCLC (Fossella et al., 2003; Chen et al., 2007).

Oral etoposide has been found to be effective in the treatment of a number of cancers (Belani et al.,1987; Williams et al., 1987; Johnson et al., 1991; Loehrer, 1992). The cellular target of etoposide is topoisomerase II, and actively dividing cells are susceptible to etoposide, especially in the S, G2, and M phases. Topoisomerase II is an important enzyme for the DNA repair; thus, inhibition of this enzyme produces synergistic effect when combined with radiotherapy.Etoposide has been administrated to treat local advanced NSCLC while combined with concurrent radiotherapy. 
Oral etoposide is not frequently used in patients with metastatic NSCLC. Although the response rate varies from 0 to $48 \%$, and toxicity is low following etoposide treatment, the median survival time (MST) of 5 months was repeatedly confirmed (Waits et al., 1992). Two phase III studies on NSCLC examined the efficacy of a platinum-based induction therapy followed immediately by maintenance therapy with non-platinum agent in the induction regimen. In both studies, patients who continued to receive the non-platinum agent as maintenance therapy had better outcomes than those without maintenance therapy (Fidias et al., 2008; PazAres et al., 2012). The present study showed oral etoposide for maintenance therapy achieved a median PFS of 5 months, which confirmed the good prognosis of these patients who experienced disease control rate following first-line chemotherapy. Since all patients had stage IV NSCLC, results obtained in our study are clinically significant. There may be a prolongation of OS, due to a longer duration of effective chemotherapy in the DC. Therefore the optimal treatment sequence and duration of chemotherapy in the DC switching to oral etoposide may have resulted in increased survival. Our results demonstrated a favorable OS and a different toxicity profile in the maintenance chemotherapy with oral etoposide. In addition, the PFS was 5 and 6 months in patients with squamous cell cancer and adenocarcinoma, respectively $(\mathrm{p}=0.38)$. Moreover, $\mathrm{a}$ fraction of patients achieved long-lasting SD and this resulted in a very interesting median time to progression. So, it was useful to prolong treatment in those patients with stable disease.

Our results show that the oral etoposide for maintenance chemotherapy had a manageable toxicity profile. In this study, all patients had stage IV NSCLC, and some of them were in low performance status and were the elderly, who may adversely influence the outcome, but they well tolerated to the treatment. On the other hand, they were all chemotherapy-naive and, therefore, likely to experience less multiple drug resistance and achieve better outcome, let alone the low incidence of toxicity when compared with other 'modern' chemotherapeutics. Finally, the cost-effectiveness of this strategy in the present study should be clearly emphasized, since even when given alone, new active drugs are more expensive than those in our regimen. On the basis of these encouraging results, evaluation of new regimens using non-cross-resistant agents delivered as maintenance therapy of NSCLC may be warranted in appropriately powered studies. This trial was statistically powered to evaluate the OS benefit of maintenance therapy in advanced NSCLC with notable exception in the delayed treatment group. Also, they are inferior to those obtained with most combination CT regimens used currently, and oral etoposide alone should, therefore, be recommended for further investi- gation. Together with mild to moderate toxicity and low cost, it may warrant further studies with large sample size to compare it with other active agents.

\section{References}

Albain KS, Crowley JJ, Turrisi AT 3rd, Gandara DR, Farrar WB, Clark JI, Beasley KR, Livingston RB. Concurrent cisplatin, etoposide, and chest radiotherapy in pathologic stage IIIB non-small-cell lung cancer: A Southwest Oncology Group phase II study, SWOG 9019. J Clin Oncol. 2002; 20: 3454-60.

Belani CP, Doyle LA, Aisner J. Etoposide: Current status and future perspectives in the management of malignant neoplasms. Cancer Chemother Pharmacol. 1994; 34: S118-26.

Brodowicz T, Krzakowski M, Zwitter M, Tzekova V, Ramlau R, Ghilezan N, Ciuleanu T, Cucevic B, Gyurkovits K, Ulsperger E, Jassem J, Grgic M, Saip P, Szilasi M, Wiltschke C, Wagnerova M, Oskina N, Soldatenkova V, Zielinski C, Wenczl M. Central European Cooperative Oncology Group CECOG. Cisplatin and gemcitabine first-line chemotherapy followed by maintenance gemcitabine or best supportive care in advanced non-small cell lung cancer: A phase III trial. Lung Cancer. 2006; 52: 155-63.

Chemotherapy in non-small cell lung cancer: A meta-analysis using updated data on individual patients from 52 randomized clinical trials. Non-small Cell Lung Cancer Collaborative Group. BMJ. 1995; 311: 899-909.

Chen YM, Perng RP, Shih JF, Tsai CM, Whang-Peng J. A randomized phase II study of docetaxel or vinorelbine in combination with cisplatin against inoperable, chemo-naive non-small-cell lung cancer in Taiwan. Lung Cancer, 2007; 56: 363-69.

Fidias PM, Dakhil SR, Lyss AP, Loesch DM, Waterhouse DM, Bromund JL, Chen R, Hristova-Kazmierski M, Treat J, Obasaju CK, Marciniak M, Gill J, Schiller JH. Phase III study of immediate compared with delayed docetaxel after frontline therapy with gemcitabine plus carboplatin in advanced non-small-cell lung cancer. J Clin Oncol. 2009; 27: 591-98.

Fossella F, Pereira JR, von Pawel J, Pluzanska A, Gorbounova V, Kaukel E, Mattson KV, Ramlau R, Szczesna A, Fidias P, Millward M, Belani CP. Randomized, multinational, phase III study of docetaxel plus platinum combinations versus vinorelbine plus cisplatin for advanced non-small-cell lung cancer: The TAX 326 study group. J Clin Oncol. 2003; 21: 3016-24.

Fukuoka M, Wu YL, Thongprasert S, Sunpaweravong P, Leong SS, Sriuranpong V, Chao TY, Nakagawa K, Chu DT, Saijo N, Duffield EL, Rukazenkov Y, Speake G, Jiang H, Armour AA, To KF, Yang JC, Mok TS. Biomarker analyses and final overall survival results from a phase III, randomized, openlabel, first-line study of gefitinib versus carboplatin/ paclitaxel in clinically selected patients with advanced nonsmall-cell lung cancer in Asia (IPASS). J Clin Oncol. 2011; 29: 2866-74.

Giaccone G. Epidermal growth factor receptor inhibitors in the treatment of non-small-cell lung cancer. J Clin Oncol. 2005; 


\section{3: $3235-42$.}

Giovannetti E, Mey V, Nannizzi S, Pasqualetti G, Marini L, Del Tacca M, Danesi R. Cellular and harmacogenetics foundation of synergistic interaction of pemetrexed and gemcitabine in human non-small-cell lung cancer cells. Mol Pharmacol. 2005; 68: 110-18.

Goldie JH, Coldman AJ. A mathematic model for relating the drug sensitivity of tumors to their spontaneous mutation rate. Cancer Treat Rep. 1979; 63: 1727-33.

Hirsch FR, Kabbinavar F, Eisen T, Martins R, Schnell FM, Dziadziuszko R, Richardson K, Richardson F, Wacker B, Sternberg DW, Rusk J, Franklin WA, Varella-Garcia M, Bunn PA Jr, Camidge DR. A randomized, phase II, biomarkerselected study comparing erlotinib to erlotinib intercalated with chemotherapy in first-line therapy for advanced nonsmall-cell lung cancer. J Clin Oncol. 2011; 29: 3567-73.

Jemal A, Siegel R, Ward E, Hao Y, Xu J, Thun MJ. Cancer statistics, 2009. CA Cancer J Clin. 2009; 59: 225-49.

Johnson DH, Hainsworth JD, Hande KR, Greco FA. Current status of etoposide in the management of small cell lung cancer. Cancer 1991; 67 Suppl: 231-44.

Loehrer PJ. Chronic oral etoposide: Trials at Indiana University and with the Hoosier Oncology Group. Semin Oncol. 1992; 19: 48-52.

Ohe Y, Ichinose Y, Nakagawa K, Tamura T, Kubota K, Yamamoto N, Adachi S, Nambu Y, Fujimoto T, Nishiwaki Y, Saijo N, Fukuoka M. Efficacy and safety of two doses of pemetrexed supplemented with folic acid and vitamin B12 in previously treated patients with non-small cell lung cancer. Clin Cancer Res. 2008; 14: 4206-12.

Paz-Ares L, de Marinis F, Dediu M, Thomas M, Pujol JL, Bidoli P, Molinier O, Sahoo TP, Laack E, Reck M, Corral J, Melemed S, John W, Chouaki N, Zimmermann AH, Visseren -Grul C, Gridelli C. Maintenance therapy with pemetrexed plus best supportive care versus placebo plus best supportive care after induction therapy with pemetrexed plus cisplatin for advanced non-squamous non-small-cell lung cancer (PARAMOUNT): A double-blind, phase 3, randomised controlled trial. Lancet Oncol. 2012; 13: 247-55.

Pedersen AG, Hansen HH. Etoposide (VP 16) in the treatment of lung cancer. Cancer Treat Rev. 1983; 10: 245-64.

Pirker R, Pereira JR, Szczesna A, von Pawel J, Krzakowski M, Ramlau R, Vynnychenko I, Park K, Yu CT, Ganul V, Roh JK, Bajetta E, O'Byrne K, de Marinis F, Eberhardt W, Goddemeier T, Emig M, Gatzemeier U. FLEX Study Team. Cetuximab plus chemotherapy in patients with advanced non-small-cell lung cancer (FLEX): An open-label randomized phase III trial. Lancet. 2009; 373: 1525-31.

Sandler A, Gray R, Perry MC, Brahmer J, Schiller JH, Dowlati A, Lilenbaum R, Johnson DH. Paclitaxel-carboplatin alone or with bevacizumab for non-small-cell lung cancer. N Engl J Med. 2006; 355: 2542-50.

Scagliotti GV, Parikh P, von Pawel J, Biesma B, Vansteenkiste J, Manegold C, Serwatowski P, Gatzemeier U, Digumarti R, Zukin M, Lee JS, Mellemgaard A, Park K, Patil S, Rolski J, Goksel T, de Marinis F, Simms L, Sugarman KP, Gandara D. Phase III study comparing cisplatin plus gemcitabine with cisplatin plus pemetrexed in chemotherapy-naive patients with advanced-stage non-small-cell lung cancer. J Clin Oncol. 2008; 26: 3543-51.

Schiller JH, Harrington D, Belani CP, Langer C, Sandler A, Krook J, Zhu J, Johnson DH; Eastern Co-operative Oncology Group. Comparison of four chemotherapy regimens for advanced non-small-cell lung cancer. N Engl J Med. 2002; 346: 92-98.

Waits TM, Johnson DH, Hainsworth JD, Hande KR, Thomas $\mathrm{M}$, Greco FA. Prolonged administration of oral etoposide in non-small-cell lung cancer: A phase II trial. J Clin Oncol. 1992; 10: 292-96.

Williams SD, Birch R, Einhorn LH, Irwin L, Greco FA, Loehrer PJ. Treatment of disseminated germ cell tumors with cisplatin, bleomycin, and either vinblastine or etoposide. $\mathrm{N}$ Engl J Med. 1987; 316: 1435-40. 